\title{
Tumors of the eye and ocular adnexa at the Philippine Eye Research Institute: a I0-year review
}

\author{
This article was published in the following Dove Press journal: \\ Clinical Ophthalmology \\ 9 July 2015 \\ Number of times this article has been viewed
}

\author{
Rolando Enrique D \\ Domingo ${ }^{1,2}$ \\ Lilibeth E Manganip' \\ Rolando M Castro' \\ 'Philippine Eye Research Institute, \\ National Institutes of Health, \\ University of the Philippines, \\ Manila, Philippines; ${ }^{2}$ Department of \\ Ophthalmology and Visual Sciences, \\ University of the Philippines College \\ of Medicine - Philippine General \\ Hospital, Manila, Philippines
}

Objective: We aimed to identify and describe the clinicopathologic characteristics of tumors of the eye and ocular adnexa at the Philippine Eye Research Institute.

Methods: The pathology reports of specimens submitted to the Ocular Pathology Section of the Philippine Eye Research Institute over a 10-year period (2003-2012) were reviewed, and collected data was subjected to descriptive statistical tabulation and analysis.

Results: A total of 1,551 histologically confirmed tumors were included. Of these, 254 were from the conjunctiva ( 155 benign, 99 malignant), 530 from the eyelids ( 360 benign, 170 malignant), 394 were intraocular tumors (21 benign, 373 malignant), and 373 were from the orbit (231 benign, 142 malignant). Retinoblastoma was the most common tumor of the eye and ocular adnexa in the series, accounting for $43.2 \%$ of all malignancies. Squamous cell carcinoma was the most frequent cancer of the conjunctiva, while basal cell carcinoma and sebaceous gland carcinoma were the most common eyelid malignancies. Orbital malignancies had a bimodal age distribution, with rhabdomyosarcoma being most frequent in children, while in adults, lymphoma was most common, followed by lacrimal gland carcinomas.

Conclusion: The majority of tumors of the conjunctiva, eyelids, and orbit were benign, while most intraocular tumors were malignant, with retinoblastoma being the most common.

Keywords: cancer, ophthalmic, retinoblastoma, clinical characteristics

\section{Introduction}

Cancer is an important cause of death and disability in the Philippines. Tumors of the eye and ocular adnexa comprise a small percentage of the total malignancies diagnosed and treated yearly. Ngelangel and Wang reported the eye cancer incidence in the Philippines at a low rate of 0.7 per 100,000 population - one of the least frequent site of malignancies in the human body. ${ }^{1}$ However, these are some of the most challenging cases seen by ophthalmologists and are the more serious eye conditions, leading possibly to blindness and death.

Several countries with pathology registries have published reports on the epidemiology of ophthalmologic malignancies. The information is very useful to clinicians as they diagnose and manage their patients. However, there is wide variation in the characteristics of these malignancies among varying population groups. The classic example would be the melanomas, which are reported as the most common primary intraocular tumors in Caucasians but which are rare in other populations. ${ }^{2}$

In Asian countries, such as Singapore, it was found that retinoblastomas are far more common than uveal melanomas and comprise more than half of reported eye cancer cases. ${ }^{3}$ Other studies from the region showed that nonpigmented eyelid tumors, such as basal cell carcinomas (BCC), squamous cell carcinomas (SCC), and sebaceous gland carcinomas (SGC) are much more common than malignant melanomas. ${ }^{4}$ 
Tumors of the conjunctiva are composed of a varied group of lesions that may originate from different cells. ${ }^{5}$ Race and geography are among several factors known to affect the incidence of epithelial and melanocytic lesions. In Africa, the coexistence of human immunodeficiency virus (HIV) and human papilloma virus (HPV) infection has been reported to increase the rate of ocular surface squamous neoplasia, particularly in women. ${ }^{6}$

Orbital lesions represent an even larger group of tumors. Shields et al published a review of 1,264 patients over a period of 30 years and found that benign were more common than malignant lesions but that malignancy became more likely with advanced age. ${ }^{7}$ Studies in Japan ${ }^{8}$ and India ${ }^{9}$ had similar findings, in that pediatric orbital tumors differ from adult tumors in terms of etiology and incidence. However, the frequency of particular tumors varies among the different study populations, with lymphoid tumors comprising a larger percentage of malignancies in Asians.

The Philippine Eye Research Institute (PERI) is home to the only specialized ocular pathology unit in the country. It has been in existence since 1966 and receives more than 200 specimens every year, coming mainly from the Philippine General Hospital Department of Ophthalmology and Visual Sciences (PGH DOVS) and partly from other regional hospitals.
Table I Frequency distribution of the tumors, according to location and malignancy, at the Philippine Eye Research Institute from 2003 to 2012

\begin{tabular}{ll}
\hline Location & Number (\%) \\
\hline Conjunctiva & 254 \\
Benign & $155(61.02 \%)$ \\
Malignant & $99(38.98 \%)$ \\
Eyelids & 530 \\
Benign & $360(63.16 \%)$ \\
Malignant & $170(36.84 \%)$ \\
Intraocular & 394 \\
Benign & $21(5.33 \%)$ \\
Malignant & $373(94.67 \%)$ \\
Orbit & 373 \\
Benign & $231(63.93 \%)$ \\
Malignant & $142(36.07 \%)$ \\
Total & 1,551 \\
Benign & $767(49.45 \%)$ \\
Malignant & $784(50.55 \%)$ \\
\hline
\end{tabular}

There is no published report describing the epidemiology of ophthalmologic tumors in the Philippines, and the lack of a national ocular tumor registry will not make this possible in the near future. Data from the ocular pathology registry of the PERI is the best resource at this time.

This study was designed 1) to identify the most common tumors of the eye and ocular adnexa diagnosed histologically at the PERI, and 2) to describe the clinicopathologic characteristics

Table 2 Distribution of benign tumors of the conjunctiva according to frequency, age, sex, laterality, and duration of symptoms (Philippine Eye Research Institute from 2003 to 2012)

\begin{tabular}{|c|c|c|c|c|c|}
\hline Tumor & $\begin{array}{l}\text { Number (\%) } \\
\mathbf{N}=\mathbf{I} 55\end{array}$ & $\begin{array}{l}\text { Mean age in } \\
\text { years (SD) }\end{array}$ & $\begin{array}{l}\text { Sex ratio } \\
(M: F)\end{array}$ & $\begin{array}{l}\text { Mean duration } \\
\text { of symptoms } \\
\text { in months (SD) }\end{array}$ & $\begin{array}{l}\text { Laterality (\%) } \\
\text { Right (R) } \\
\text { Left (L) } \\
\text { Bilateral (B) }\end{array}$ \\
\hline Epithelial cyst & $42(27.1)$ & $35.49(19.50)$ & $1: 1.2$ & $38.60(63.06)$ & $\begin{array}{l}\text { R } 46.2 \\
\text { L } 53.8\end{array}$ \\
\hline Dermoid & $30(19.4)$ & $19.27(|8.5|)$ & $1: 1.2$ & 125.73 (I22.04) & $\begin{array}{l}\text { R } 66.7 \\
\text { L } 29.6 \\
\text { B } 3.7\end{array}$ \\
\hline Nevus & $24(15.5)$ & $32.00(24.6 I)$ & $1: 1.9$ & $215.35(269.16)$ & $\begin{array}{l}\text { R } 50.0 \\
\text { L } 50.0\end{array}$ \\
\hline Pyogenic granuloma & $14(9.0)$ & $27.08(19.67)$ & $1: 1.6$ & $8.04(10.49)$ & $\begin{array}{l}R \text { 7I.4 } \\
L 28.6\end{array}$ \\
\hline Squamous papilloma & II (7.I) & $28.45(15.57)$ & $1.2: 1$ & I5. 14 (20.42) & $\begin{array}{l}\text { R } 40.0 \\
\text { L } 60.0\end{array}$ \\
\hline $\begin{array}{l}\text { Primary acquired } \\
\text { melanosis }\end{array}$ & $10(6.4)$ & $33.90(19.93)$ & $1.5: 1$ & $36.26(64.18)$ & $\begin{array}{l}\text { R } 55.6 \\
\text { L } 44.4\end{array}$ \\
\hline $\begin{array}{l}\text { Atypical lymphoid } \\
\text { hyperplasia }\end{array}$ & $5(3.2)$ & $36.80(19.64)$ & $1.5: 1$ & $7.50(4.43)$ & $\begin{array}{l}\text { R } 0.0 \\
\text { L } 100.0\end{array}$ \\
\hline $\begin{array}{l}\text { Reactive lymphoid } \\
\text { hyperplasia }\end{array}$ & $5(3.2)$ & $45.40(23.10)$ & $1.5: 1$ & $19.25(21.69)$ & $\begin{array}{l}\text { R } 80.0 \\
\text { L } 20.0\end{array}$ \\
\hline Sebaceous hyperplasia & $3(1.9)$ & $25.33(19.14)$ & $2: 1$ & $64.03(73.28)$ & $\begin{array}{l}\text { R } 100.0 \\
\text { L } 0.0\end{array}$ \\
\hline Others & II (7.I) & & & & \\
\hline
\end{tabular}

Abbreviation: SD, standard deviation. 
of the tumors in terms of clinical history and diagnosis, and distribution in terms of age, sex, and location of the lesion. This information will be useful to clinicians, patients, students, health care administrators, and service providers.

\section{Materials and methods}

This was a health records review study approved by the University of the Philippines National Institutes of Health Research Ethics Board.

All requests for histopathologic studies with accompanying specimens submitted to the PERI Ocular Pathology Section from January 1, 2003 to December 31, 2012 were examined. The final reports issued for the specimens, containing the description and final diagnosis, were also reviewed.

All specimens with a histopathologic diagnosis of a benign or malignant neoplastic lesion were included. In case of repeat submissions of a specimen from the same patient within the study period, from the same or recurrent lesion, only the first submission was included.

A data collection form was completed for each sample. The information included the specimen number, age, sex, location of the lesion, description of the lesion, short medical history, clinical diagnosis, and histopathologic diagnosis. The name of the patient was not recorded, and identity was kept confidential.

The reports were separated into four categories depending on the location of the tumor: conjunctiva, intraocular, eyelid, and orbit. All information was then subjected to descriptive statistical collation and analysis using the open-access software Epi Info ${ }^{\mathrm{TM}}$ 3.5.4 (Centers for Disease Control and Prevention, Atlanta, GA, USA).

\section{Results}

A total of 1,551 histologically confirmed tumors were included in the study. These were subdivided into four categories: conjunctiva, eyelid, intraocular, and orbit.

Tumors classified under conjunctiva were lesions from the bulbar conjunctiva, fornix, and limbus. The eyelid tumors were located anywhere through the whole thickness of the upper and lower lids, including the medial and lateral canthus areas. Orbital lesions involved the soft tissues of the orbit, the lacrimal gland, lacrimal sac, extraocular muscles, and optic nerve. The intraocular tumors were lesions of the uveal tract and the retina.

The majority (above 60\%) of conjunctiva, eyelid, and orbit tumors were benign. On the other hand, the great majority of intraocular tumors were malignant. Considering all the tumors together, the number of benign and malignant tumors was almost equal (Table 1).

Benign lesions comprised $61.02 \%$ of conjunctival tumors, the most common of which were epithelial cysts, dermoids, and nevi (Table 2). As expected, dermoids as well as the other benign lesions came from younger patients and had a long duration of symptoms, except for the conditions with inflammatory components, such as pyogenic granuloma.

SCC accounted for $40.4 \%$ of all malignant conjunctival tumors, followed by its precursor, conjunctival intraepithelial neoplasia (CIN), with $26.3 \%$. The other important malignancies were melanoma and lymphoma, and the four lesions together comprised $97 \%$ of all conjunctival cancers and precancers (Table 3 ). The patients with malignant lesions were older than those with benign lesions and were mostly male. The mean duration of symptoms for malignant lesions

Table 3 Distribution of malignant and premalignant tumors of the conjunctiva according to frequency, age, sex, laterality, and duration of symptoms (Philippine Eye Research Institute from 2003 to 2012)

\begin{tabular}{|c|c|c|c|c|c|}
\hline Tumor & $\begin{array}{l}\text { Number (\%) } \\
\mathbf{N}=99\end{array}$ & $\begin{array}{l}\text { Mean age in } \\
\text { years (SD) }\end{array}$ & $\begin{array}{l}\text { Sex ratio } \\
(M: F)\end{array}$ & $\begin{array}{l}\text { Mean duration } \\
\text { of symptoms } \\
\text { in months (SD) }\end{array}$ & $\begin{array}{l}\text { Laterality (\%) } \\
\text { Right (R) } \\
\text { Left (L) } \\
\text { Bilateral (B) }\end{array}$ \\
\hline Squamous cell carcinoma & $40(40.4)$ & $62.38(15.40)$ & I.7:1 & $13.72(2 \mid .05)$ & $\begin{array}{l}\text { R } 52.8 \\
\text { L } 47.2\end{array}$ \\
\hline $\begin{array}{l}\text { Conjunctival intraepithelial } \\
\text { neoplasia }\end{array}$ & $26(26.3)$ & $56.46(18.66)$ & $3.2: 1$ & $12.23(13.93)$ & $\begin{array}{l}\text { R } 26.1 \\
L 73.9\end{array}$ \\
\hline Melanoma & $18(18.2)$ & $48.89(17.11)$ & I.6:1 & 63.86 (I32.59) & $\begin{array}{l}\text { R } 66.7 \\
\text { L } 33.3\end{array}$ \\
\hline Lymphoma & $12(12.1)$ & $45.50(16.43)$ & $2: 1$ & $16.94(16.86)$ & $\begin{array}{l}R 72.7 \\
L 18.2 \\
\text { B } 9.1\end{array}$ \\
\hline Basal cell carcinoma & $2(2.0)$ & $60.00(0.00)$ & $2: 0$ & $6.50(7.78)$ & $\begin{array}{l}\text { R } 100.0 \\
\text { L } 0.0\end{array}$ \\
\hline Sebaceous gland carcinoma & I (I.0) & $46.00(0.00)$ & $\mathrm{I}: 0$ & $24.00(0.00)$ & $\begin{array}{l}\text { R } 100.0 \\
\text { L } 0.0\end{array}$ \\
\hline
\end{tabular}

Abbreviation: SD, standard deviation. 
Table 4 Distribution of benign eye lid tumors according to frequency, age, sex, laterality, and duration of symptoms (Philippine Eye Research Institute from 2003 to 2012)

\begin{tabular}{|c|c|c|c|c|c|}
\hline Tumor & $\begin{array}{l}\text { Number (\%) } \\
\mathbf{N}=360\end{array}$ & $\begin{array}{l}\text { Mean age in } \\
\text { years (SD) }\end{array}$ & $\begin{array}{l}\text { Sex ratio } \\
(M: F)\end{array}$ & $\begin{array}{l}\text { Mean duration } \\
\text { of symptoms } \\
\text { in months (SD) }\end{array}$ & $\begin{array}{l}\text { Laterality (\%) } \\
\text { Right (R) } \\
\text { Left (L) } \\
\text { Bilateral (B) }\end{array}$ \\
\hline Epidermoid cysts & $116(32.2)$ & $31.75(21.48)$ & $\mathrm{I}: \mathrm{I}$ & 55.17 (79.98) & $\begin{array}{l}\text { R } 55.7 \\
\text { L } 43.5 \\
\text { B } 0.9\end{array}$ \\
\hline Nevus & $71(19.7)$ & $43.66(16.96)$ & $\mathrm{I}: 4$ & $215.86(182.47)$ & $\begin{array}{l}\text { R } 51.5 \\
\text { L } 48.5\end{array}$ \\
\hline Squamous papilloma & $70(19.4)$ & $40.09(20.22)$ & $1: 1.9$ & $45.05(77.77)$ & $\begin{array}{l}\text { R } 46.4 \\
\text { L } 47.8 \\
\text { B } 5.8\end{array}$ \\
\hline Neurofibroma & $17(4.7)$ & $18.12(12.7)$ & $\mathrm{I}: 1.8$ & $\mid 46.38(|| 8.2 \mid)$ & $\begin{array}{l}\text { R } 64.7 \\
\text { L } 29.4 \\
\text { B } 5.9\end{array}$ \\
\hline Seborrheic keratosis & $12(3.3)$ & $62.58(14.38)$ & $\mathrm{I}: 3$ & $93.82(198.95)$ & $\begin{array}{l}\text { R } 36.4 \\
\text { L } 36.6 \\
\text { B } 27.3\end{array}$ \\
\hline Chalazion & $10(2.8)$ & $35.70(16.76)$ & $1.5: 1$ & $9.06(7.61)$ & $\begin{array}{l}\text { R } 55.6 \\
\text { L } 44.4\end{array}$ \\
\hline Xanthelasma & $8(2.2)$ & $52.00(7.14)$ & $0: 8$ & 21.07 (19.89) & $\begin{array}{l}\text { R } 42.9 \\
\text { L } 28.6 \\
\text { B } 28.6\end{array}$ \\
\hline Hemangioma & $7(1.9)$ & $31.29(17.62)$ & $0: 7$ & 87.83 (I22.84) & $\begin{array}{l}\text { R } 50.0 \\
\text { L } 50.0\end{array}$ \\
\hline Lymphoid hyperplasia & $7(1.9)$ & $45.14(12.58)$ & $\mathrm{I}: 2.5$ & $49.00(100.18)$ & $\begin{array}{l}\text { R } 28.6 \\
\text { L } 71.4\end{array}$ \\
\hline Schwannoma & $7(1.9)$ & $36.00(12.17)$ & $\mathrm{I}: 1.3$ & $25.50(46.53)$ & $\begin{array}{l}\text { R } 100.0 \\
\text { L } 0.0\end{array}$ \\
\hline Pyogenic granuloma & $6(1.7)$ & $19.00(22.34)$ & $2: 1$ & $5.17(6.05)$ & $\begin{array}{l}\text { R } 60.0 \\
\text { L } 40.0\end{array}$ \\
\hline Lymphangioma & $5(1.4)$ & $37.80(27.20)$ & $4: 1$ & $21.10(18.00)$ & $\begin{array}{l}\text { R } 60.0 \\
\text { L } 40.0\end{array}$ \\
\hline Lipoma & $4(1.1)$ & $31.50(13.53)$ & $\mathrm{I}: 3$ & $12.00(0.00)$ & $\begin{array}{l}\text { R } 75.0 \\
\text { L } 25.0\end{array}$ \\
\hline Benign sweat gland tumors & $4(1.1)$ & $40.50(12.37)$ & $1: 3$ & I 59.50 (293.72) & $\begin{array}{l}\text { R } 50.0 \\
\text { L } 50.0\end{array}$ \\
\hline Benign pilar structure tumors & $3(0.8)$ & $56.67(14.29)$ & $\mathrm{I}: 2$ & $25.33(30.29)$ & $\begin{array}{l}\text { R } 66.7 \\
\text { L } 33.3\end{array}$ \\
\hline Xanthogranuloma & $3(0.8)$ & $23.33(4.62)$ & $2: 1$ & I5.33 (I8.33) & $\begin{array}{l}\text { R } 66.7 \\
\text { L } 33.3\end{array}$ \\
\hline Others & $10(2.8)$ & & & & \\
\hline
\end{tabular}

Abbreviation: SD, standard deviation.

was shorter compared with benign tumors, with the exception of conjunctival melanomas.

Benign eyelid tumors came in a wide variety due to its inherent anatomy (Table 4). These were predominantly in females, and the most common lesions were epidermoid cysts, nevi, and papillomas. Neurofibromas were seen in young patients (and mostly underwent tumor debulking), while seborrheic keratosis came from older patients. These five comprised $79.3 \%$ of the total.

$\mathrm{BCC}$ and SGC were tied as the most frequent malignant tumors of the eyelid (30.6\%) and were both seen in slightly more women than men. SCC, melanoma, and lymphoma completed the top five, to account for $96.6 \%$ of the total, with the mean age of patients ranging from 53.64 to 63.23 years (Table 5). BCC and melanomas were more common in the lower lid while SGC and SCC were more common in the upper lid.

There were few cases of enucleated eyes with benign lesions. These were mostly from children suspected of having retinoblastoma, and the most common mimicking tumor was retinal dysplasia (Table 6). There were three cysts and one ciliary body leiomyoma excised with the suspicion of a uveal melanoma. 
Table 5 Distribution of malignant eye lid tumors according to frequency, age, sex, laterality, and duration of symptoms (Philippine Eye Research Institute from 2003 to 2012)

\begin{tabular}{|c|c|c|c|c|c|}
\hline Tumor & $\begin{array}{l}\text { Number (\%) } \\
\mathbf{N}=170\end{array}$ & $\begin{array}{l}\text { Mean age in } \\
\text { years (SD) }\end{array}$ & $\begin{array}{l}\text { Sex ratio } \\
(M: F)\end{array}$ & $\begin{array}{l}\text { Mean duration } \\
\text { of symptoms in } \\
\text { months (SD) }\end{array}$ & $\begin{array}{l}\text { Laterality (\%) } \\
\text { Right (R) } \\
\text { Left (L) } \\
\text { Bilateral (B) }\end{array}$ \\
\hline Basal cell carcinoma & $52(30.6)$ & $63.23(12.52)$ & $\mathrm{I}: 1.3$ & $84.57(117.08)$ & $\begin{array}{l}\text { R } 52.0 \\
\text { L } 48.0\end{array}$ \\
\hline Sebaceous gland carcinoma & $52(30.6)$ & $58.14(13.52)$ & $\mathrm{I}: 1.3$ & $32.40(89.88)$ & $\begin{array}{l}\text { R } 53.8 \\
\text { L } 46.2\end{array}$ \\
\hline Squamous cell carcinoma & $29(17.1)$ & $59.14(17.74)$ & $\mathrm{I}: 3$ & $23.0(22.7)$ & $\begin{array}{l}\text { R } 38.5 \\
\text { L } 61.5\end{array}$ \\
\hline Melanoma & $20(11.8)$ & $56.75(14.62)$ & $1.9: 1$ & $22.88(28.91)$ & $\begin{array}{l}\text { R } 52.6 \\
\text { L } 47.4\end{array}$ \\
\hline Lymphoma & II (6.5) & $53.64(12.02)$ & $1.2: 1$ & $43.25(60.17)$ & $\begin{array}{l}\text { R } 33.3 \\
\text { L } 66.7\end{array}$ \\
\hline Malignant pilar structure tumors & $2(1.2)$ & $83.00(11.31)$ & $2: 0$ & $25.33(30.29)$ & $\begin{array}{l}\text { R } 50.0 \\
\text { L } 50.0\end{array}$ \\
\hline Metastatic carcinoma & $2(1.2)$ & $48.00(4.24)$ & $0: 2$ & $12.00(0.00)$ & $\begin{array}{l}\text { R } 50.0 \\
\text { L } 50.0\end{array}$ \\
\hline Mucoepidermoid carcinoma & $\mathrm{I}(0.6)$ & $28.00(0.00)$ & $\mathrm{I}: 0$ & $24.00(0.00)$ & $\begin{array}{l}\text { R } 100.0 \\
L 0.0\end{array}$ \\
\hline Retinoblastoma & I (0.6) & $14.00(0.00)$ & $0: 1$ & $8.00(0.00)$ & $\begin{array}{l}\text { R } 100.0 \\
\text { L } 0.0\end{array}$ \\
\hline
\end{tabular}

Abbreviation: SD, standard deviation.

Retinoblastoma was the most common malignant tumor inside the eye and all the ocular adnexa. It accounted for $90.9 \%$ of all intraocular cancers and $43.24 \%$ of all malignant tumors in this study. In adults, uveal melanoma was the most common intraocular cancer found in enucleated eyes, followed by metastatic carcinoma (Table 7).

Due to the large variety of tissues present in the orbit, there was a long list of histologic diagnoses. The most common were hemangiomas, dermoids, and benign mixed tumor of the lacrimal gland. The ten most common comprised $86.7 \%$ of the total (Table 8 ). The patients with lymphoid hyperplasia were the oldest, with a mean age of 50.80 years.

The most common malignant tumors of the orbit were lymphoma and cancers of the lacrimal gland in adults, and rhabdomyosarcoma in children (Table 9). Tumors that invaded the orbit secondarily in late-stage disease, such as SCC, retinoblastoma, SGC, and $\mathrm{BCC}$, were still quite

Table 6 Distribution of benign intraocular tumors according to frequency, age, sex, laterality, and duration of symptoms (Philippine Eye Research Institute from 2003 to 2012)

\begin{tabular}{|c|c|c|c|c|c|}
\hline Tumor & $\begin{array}{l}\text { Number (\%) } \\
\mathbf{N}=\mathbf{2} \text { I }\end{array}$ & $\begin{array}{l}\text { Mean age in } \\
\text { years (SD) }\end{array}$ & $\begin{array}{l}\text { Sex ratio } \\
(M: F)\end{array}$ & $\begin{array}{l}\text { Mean duration } \\
\text { of symptoms } \\
\text { in months (SD) }\end{array}$ & $\begin{array}{l}\text { Laterality (\%) } \\
\text { Right (R) } \\
\text { Left (L) } \\
\text { Bilateral (B) }\end{array}$ \\
\hline Retinal dysplasia & $9(42.9)$ & $2.09(2.4 I)$ & $2: 1$ & $7.71(9.20)$ & $\begin{array}{l}\text { R } 44.4 \\
\text { L } 44.4 \\
\text { B II.I }\end{array}$ \\
\hline Epithelial cyst & $3(14.3)$ & $29.00(31.11)$ & $1: 2$ & $24.00(0.00)$ & $\begin{array}{l}\text { R } 0.0 \\
\text { L } 00.0\end{array}$ \\
\hline Coats disease & $2(9.5)$ & $2.00(2.00)$ & $2: 0$ & $5.00(4.24)$ & $\begin{array}{l}\text { R } 00.0 \\
\text { L } 0.0\end{array}$ \\
\hline Choroidal hemangioma & $2(9.5)$ & $15.50(13.43)$ & $\mathrm{I}: \mathrm{I}$ & $6.25(8.13)$ & $\begin{array}{l}\text { R } 50.0 \\
\text { L } 50.0\end{array}$ \\
\hline Granuloma, unspecified & $2(9.5)$ & $2.50(0.7 I)$ & $\mathrm{I}: \mathrm{I}$ & $14.00(14.14)$ & $\begin{array}{l}\text { R } 00.0 \\
\text { L } 0.0\end{array}$ \\
\hline Retinocytoma & $2(9.5)$ & $27.00(35.35)$ & $\mathrm{I}: \mathrm{I}$ & $5.00(2.83)$ & $\begin{array}{l}\text { R } 50.0 \\
\text { L } 50.0\end{array}$ \\
\hline Ciliary body leiomyoma & I (4.8) & $29.00(0.00)$ & $0: 1$ & $5.00(0.00)$ & $\begin{array}{l}\text { R } 100.0 \\
\text { L } 0.0\end{array}$ \\
\hline
\end{tabular}

Abbreviation: SD, standard deviation. 
Table 7 Distribution of malignant intraocular tumors according to frequency, age, sex, laterality, and duration of symptoms (Philippine Eye Research Institute from 2003 to 2012)

\begin{tabular}{|c|c|c|c|c|c|}
\hline Tumor & $\begin{array}{l}\text { Number (\%) } \\
\mathbf{N}=373\end{array}$ & $\begin{array}{l}\text { Mean age in } \\
\text { years (SD) }\end{array}$ & $\begin{array}{l}\text { Sex ratio } \\
(M: F)\end{array}$ & $\begin{array}{l}\text { Mean duration } \\
\text { of symptoms } \\
\text { in months (SD) }\end{array}$ & $\begin{array}{l}\text { Laterality (\%) } \\
\text { Right (R) } \\
\text { Left (L) } \\
\text { Bilateral (B) }\end{array}$ \\
\hline \multirow[t]{3}{*}{ Retinoblastoma } & $339(90.9)$ & $2.45(1.86)$ & $1.1: 1$ & $10.61(11.34)$ & R 38.8 \\
\hline & & & & & L 38.2 \\
\hline & & & & & B 23.0 \\
\hline \multirow[t]{2}{*}{ Uveal melanoma } & $30(8.0)$ & $48.37(13.86)$ & I:I.4 & I4.74 (23.09) & R 44.8 \\
\hline & & & & & L 55.2 \\
\hline \multirow[t]{2}{*}{ Metastatic carcinoma } & $4(1.1)$ & $52.33(19.14)$ & $\mathrm{I}: \mathrm{I}$ & $4.67(2.08)$ & R 75.0 \\
\hline & & & & & L 25.0 \\
\hline
\end{tabular}

Abbreviation: SD, standard deviation.

common in our setting (18.9\%). Many other rare and unusual malignancies were seen in the list.

\section{Discussion}

After reviewing the records of all tumors at the PERI ocular pathology laboratory for a period of 10 years, we found that the majority $(64.8 \%)$ of tumors from the conjunctiva, eyelids, and orbit were benign. This is a common finding in studies from Hong Kong, ${ }^{4}$ Japan, ${ }^{8}$ USA, ${ }^{7}$ and Spain. ${ }^{5}$ In contrast, $94.67 \%$ of intraocular tumors were malignant. In our clinics, an eye would not be enucleated unless there is very strong evidence of cancer and there is no hope for vision.

The initial diagnosis indicated on the referral form was consistent with the final histopathologic diagnosis in $68.4 \%$

Table 8 Distribution of benign tumors of the orbit according to frequency, age, sex, laterality, and duration of symptoms (Philippine Eye Research Institute from 2003 to 2012)

\begin{tabular}{|c|c|c|c|c|c|}
\hline Tumor & $\begin{array}{l}\text { Number (\%) } \\
\mathbf{N}=23 \text { I }\end{array}$ & $\begin{array}{l}\text { Mean age in } \\
\text { years (SD) }\end{array}$ & $\begin{array}{l}\text { Sex ratio } \\
(M: F)\end{array}$ & $\begin{array}{l}\text { Mean duration } \\
\text { of symptoms } \\
\text { in months (SD) }\end{array}$ & $\begin{array}{l}\text { Laterality (\%) } \\
\text { Right (R) } \\
\text { Left (L) }\end{array}$ \\
\hline Hemangioma & $38(16.5)$ & $34.92(17.73)$ & $1: 1.9$ & $47.54(60.05)$ & $\begin{array}{l}\text { R } 63.9 \\
\text { L 36. }\end{array}$ \\
\hline Dermoid & $34(14.7)$ & $20.34(18.30)$ & $\mathrm{I}: \mathrm{I}$ & $120.53(155.32)$ & $\begin{array}{l}\text { R } 46.9 \\
\text { L } 53.1\end{array}$ \\
\hline Benign mixed tumor & $26(11.3)$ & $34.85(15.29)$ & $1: 1.9$ & $22.56(21.39)$ & $\begin{array}{l}\text { R } 45.8 \\
\text { L } 54.2\end{array}$ \\
\hline Schwannoma & $21(9.1)$ & $44.45(15.70)$ & $\mathrm{I}: 2.5$ & $28.60(31.92)$ & $\begin{array}{l}\text { R } 66.7 \\
\text { L } 33.3\end{array}$ \\
\hline Neurofibroma & I8 (7.8) & $27.00(17.75)$ & $\mathrm{I}: 1.4$ & $42.69(52.45)$ & $\begin{array}{l}\text { R 4I.2 } \\
\text { L } 58.8\end{array}$ \\
\hline Lymphoid hyperplasia & $16(6.9)$ & $50.80(18.66)$ & 4.3:1 & I3.63 (I3.80) & $\begin{array}{l}\text { R } 43.8 \\
\text { L } 56.2\end{array}$ \\
\hline Lymphangioma & $14(6.1)$ & $19.38(12.60)$ & $\mathrm{I}: 1.3$ & $54.57(65.37)$ & $\begin{array}{l}\text { R } 46.2 \\
\text { L } 53.8\end{array}$ \\
\hline Optic nerve meningioma & $14(6.1)$ & $32.7 \mid(2 \mid .56)$ & $\mathrm{I}: 1.8$ & $26.33(20.72)$ & $\begin{array}{l}\text { R } 46.2 \\
\text { L } 53.8\end{array}$ \\
\hline Non-optic nerve meningioma & $10(4.3)$ & $47.5(14.33)$ & 2.3:1 & $42.40(50.57)$ & $\begin{array}{l}\text { R } 30.0 \\
\text { L } 70.0\end{array}$ \\
\hline Pseudotumor & $9(3.9)$ & $35.89(15.50)$ & $\mathrm{I}: 3.4$ & $22.56(21.39)$ & $\begin{array}{l}\text { R } 33.3 \\
\text { L } 66.7\end{array}$ \\
\hline Optic nerve glioma & $7(3.0)$ & $14.29(13.21)$ & I.3:1 & $14.50(10.58)$ & $\begin{array}{l}\text { R } 28.6 \\
\text { L 7I.4 }\end{array}$ \\
\hline Granuloma, unspecified & $4(1.7)$ & $30.00(26.04)$ & $3: 1$ & $33.67(53.98)$ & $\begin{array}{l}\text { R } 50.0 \\
\text { L } 50.0\end{array}$ \\
\hline Lipoma & $4(1.7)$ & $25.25(7.80)$ & $0: 4$ & $21.00(11.49)$ & $\begin{array}{l}\text { R } 66.7 \\
\text { L } 33.3\end{array}$ \\
\hline Osteoma & $3(1.3)$ & $32.67(1.58)$ & $3: 0$ & $21.00(2 \mid .2 I)$ & $\begin{array}{l}\text { R } 0.0 \\
\text { L } 100.0\end{array}$ \\
\hline Others & I $3(5.6)$ & $*$ & $*$ & $*$ & $*$ \\
\hline
\end{tabular}

Note: *Uncommon lesions, data cannot be grouped.

Abbreviation: SD, standard deviation. 
Table 9 Distribution of malignant tumors of the orbit according to frequency, age, sex, laterality, and duration of symptoms (Philippine Eye Research Institute from 2003 to 2012)

\begin{tabular}{|c|c|c|c|c|c|}
\hline Tumor & $\begin{array}{l}\text { Number (\%) } \\
N=142\end{array}$ & $\begin{array}{l}\text { Mean age in } \\
\text { years (SD) }\end{array}$ & $\begin{array}{l}\text { Sex ratio } \\
(M: F)\end{array}$ & $\begin{array}{l}\text { Mean duration } \\
\text { of symptoms } \\
\text { in months (SD) }\end{array}$ & $\begin{array}{l}\text { Laterality (\%) } \\
\text { Right (R) } \\
\text { Left (L) } \\
\text { Bilateral (B) }\end{array}$ \\
\hline \multirow[t]{3}{*}{ Lymphoma } & $36(25.4)$ & $55.51(17.65)$ & I.4:I & $22.12(26.36)$ & R 41.2 \\
\hline & & & & & L 55.9 \\
\hline & & & & & В 2.9 \\
\hline \multirow[t]{2}{*}{ Adenoid cystic carcinoma } & $23(16.2)$ & $34.26(18.04)$ & I.3:1 & $13.95(9.98)$ & R 38.9 \\
\hline & & & & & L 6I.I \\
\hline \multirow[t]{2}{*}{ Rhabdomyosarcoma } & $12(8.5)$ & $6.02(4.68)$ & $2: 1$ & $7.00(8.46)$ & $\mathrm{R} 4 \mathrm{I} .7$ \\
\hline & & & & & L 58.3 \\
\hline \multirow[t]{2}{*}{ Malignant mixed tumor } & $10(7.0)$ & $45.89(16.25)$ & $\mathrm{I}: \mathrm{I}$ & $39.10(43.46)$ & R 70.0 \\
\hline & & & & & L 30.0 \\
\hline \multirow[t]{2}{*}{ Squamous cell carcinoma } & $7(4.9)$ & $45.00(6.38)$ & $2.5: 1$ & $13.14(12.31)$ & R 50.0 \\
\hline & & & & & L 50.0 \\
\hline \multirow[t]{2}{*}{ Retinoblastoma } & $7(4.9)$ & $4.7 \mathrm{I}(3.45)$ & $1.3: 1$ & $6.82(4.96)$ & R 85.7 \\
\hline & & & & & L 14.3 \\
\hline \multirow{2}{*}{ Sebaceous gland carcinoma } & $7(4.9)$ & $54.80(20.62)$ & 2.3:1 & $23.17(28.79)$ & R 100.0 \\
\hline & & & & & L 0.0 \\
\hline \multirow[t]{2}{*}{ Metastatic carcinoma } & $6(4.2)$ & $49.67(24.69)$ & $2: 1$ & $7.20(3.63)$ & R 40.0 \\
\hline & & & & & L 60.0 \\
\hline \multirow[t]{2}{*}{ Undifferentiated tumor } & $6(4.2)$ & $25.83(29.76)$ & $2: 1$ & $25.75(3 \mathrm{I} .6 \mathrm{I})$ & R 66.7 \\
\hline & & & & & L 33.3 \\
\hline \multirow[t]{2}{*}{ Basal cell carcinoma } & $6(4.2)$ & $66.00(9.65)$ & $1: 5$ & $68.00(45.32)$ & R 50.0 \\
\hline & & & & & L 50.0 \\
\hline \multirow[t]{2}{*}{ Adenocarcinoma of lacrimal gland } & $5(3.5)$ & $57.80(16.00)$ & $4: 1$ & $8.58(\mid 3.37)$ & R 60.0 \\
\hline & & & & & L 40.0 \\
\hline \multirow[t]{2}{*}{ Epithelial carcinoma lacrimal sac } & $3(2.1)$ & $39.33(5.86)$ & $2: 1$ & 15.67 (I7.67) & R 50.0 \\
\hline & & & & & L 50.0 \\
\hline \multirow[t]{2}{*}{ Hemangiopericytoma } & $3(2.1)$ & $20.67(8.08)$ & $2: 1$ & $15.00(7.94)$ & R 33.3 \\
\hline & & & & & L 66.7 \\
\hline \multirow[t]{2}{*}{ Granular cell tumor } & $2(1.4)$ & $51.00(0.00)$ & $2: 0$ & $36.00(0.00)$ & R 0.0 \\
\hline & & & & & L 100.0 \\
\hline \multirow[t]{2}{*}{ Neuroblastoma } & $2(1.4)$ & $13.50(\mid 7.68)$ & $0: 2$ & $4.75(6.01)$ & R 50.0 \\
\hline & & & & & L 50.0 \\
\hline \multirow[t]{2}{*}{ Primitive neuroectodermal tumor } & $2(1.4)$ & $18.00(5.66)$ & $0: 2$ & NR & R 50.0 \\
\hline & & & & & L 50.0 \\
\hline \multirow[t]{2}{*}{ Angiosarcoma } & I (0.7) & $54.00(0.00)$ & $0: 1$ & NR & $\mathrm{R} 0.0$ \\
\hline & & & & & L 00.0 \\
\hline \multirow[t]{2}{*}{ Liposarcoma } & $\mathrm{I}(0.7)$ & $69.00(0.00)$ & $0: 1$ & $3.00(0.00)$ & $\mathrm{R} 0.0$ \\
\hline & & & & & L 100.0 \\
\hline \multirow[t]{2}{*}{ Osteosarcoma } & $\mathrm{I}(0.7)$ & $44.00(0.00)$ & $0: 1$ & NR & $\mathrm{R} 100.0$ \\
\hline & & & & & L 0.0 \\
\hline \multirow[t]{2}{*}{ Sarcoma, unspecified } & I (0.7) & $11.00(0.00)$ & $0: 1$ & $1.50(0.00)$ & $\mathrm{R} 100.0$ \\
\hline & & & & & L 0.0 \\
\hline Plasmacytoma & I (0.7) & $50.00(0.00)$ & $0: 1$ & NR & NR \\
\hline
\end{tabular}

Abbreviations: NR, not reported; SD, standard deviation.

tumors of the conjunctiva, $67.8 \%$ of the eyelids, $94.4 \%$ of intraocular lesions, and $50.3 \%$ of the orbit. The conjunctiva and eyelids are similar in the ease of visualization and examination of lesions. The accuracy of diagnosis of intraocular tumors with the use of the indirect ophthalmoscope and imaging procedures, such as ultrasound, computerized tomography, and magnetic resonance imaging, are well documented. ${ }^{10}$ Tumors of the orbit are the most difficult to diagnose clinically due to the wide variety of lesions, and the difficulty of examination and limitation in the accuracy of imaging. Of the orbital specimens submitted, $25.5 \%$ had the clinical diagnosis listed as "unspecified mass".

Retinoblastoma was the most common malignancy. This has been a common finding in several reports, especially in developing countries. ${ }^{3}$ Clinically, it is also the most significant because it affects young children and leads to blindness and death if untreated. The number of patients seen and operated on at the PGH DOVS was very 
consistent over the study period, and this pattern is not likely to change.

The mean age at surgery was 2.45 years, and the mean duration of symptoms (primarily leukocoria) was 10.61 months. This means that diagnosis and treatment was delayed, and most of these patients, of whom $23.0 \%$ were bilateral, were enucleated at an advanced stage of disease. Of all the children enucleated with a clinical diagnosis of retinoblastoma, 95.7\% were histologically proven correct and only $4.3 \%$ turned out to be pseudoretinoblastomas. This rate of clinical accuracy is comparable with the best centers in the world. ${ }^{10}$

In adults, uveal melanomas were the most common intraocular malignancies seen. Melanomas are much more prevalent in Caucasian populations and are rare in more pigmented individuals. In our series, there was an average of three cases per year with a mean patient age of 48.37 years and slight predominance in females. This is different from reports from other countries. ${ }^{2}$ In the Armed Forces Institute of Pathology (AFIP) registry the patients had a median age of 53 years and were predominantly male; ${ }^{11}$ racial differences and variations in natural pigmentation may be the reason for the discrepancy.

It is a consensus among all reports that the four most common malignancies involving the eyelids are BCC, SCC, $\mathrm{SGC}$, and melanoma; however their relative frequencies differ. BCC is usually the most common and accounts for more than $80 \%$ of eyelid malignancies in Singapore ${ }^{12}$ and the USA, ${ }^{13}$ probably due to high excision rates for suspicious skin lesions. Our finding of $30.6 \%$ is similar to reports from Japan $^{14}$ and India. ${ }^{15}$

SGC are relatively rare in Caucasian populations but are more common in Asians. In our series SGC was tied with $\mathrm{BCC}$ at $30.6 \%$ - a figure similar to those reported in India and People's Republic of China. ${ }^{16}$ It was more common in women in our series, similar to other reports from Asia. ${ }^{4,12}$

The proportion of malignant melanomas seen was surprisingly high, at $11.8 \%$ of all eyelid cancers. This is a condition more commonly seen in Caucasians and less in pigmented people. The seemingly disproportionate number of melanomas may be due to the fact that the PGH DOVS is a referral center for difficult cases from all over the country, and affected patients are sent there by ophthalmologists who lack experience with the condition. In the same vein, the number of BCC might have been small because these are slow-growing, nonaggressive tumors that are tolerated by patients and can be managed at primary and secondary centers.

The most common site of SCC in the eye and ocular adnexa was the conjunctiva, and this was confirmed by several reports. In our series, SCC accounted for $40.4 \%$ of all conjunctival malignancies and was seen in patients with a mean age of 62.48 years. Almost twice as many men were affected, probably due to increased occupational sun exposure. The next lesion most commonly diagnosed $(26.3 \%)$ histologically was CIN, the premalignant precursor of SCC, with patients having a younger mean age of 56.46 years, as expected. Less than a quarter of the CIN patients were women, and SCC and CIN together comprised more than two-thirds of all malignant and premalignant lesions of the conjunctiva.

Melanomas were the third most common malignant conjunctival tumors, at $18.2 \%$. This is similar to other Asian reports but lower than that seen in Caucasian populations, where light pigmentation is risk factor for developing melanoma. ${ }^{3,5}$ Lymphoma is the other important conjunctival malignancy. Due to the presence of lymphocytes in the substantia propria, $70 \%-80 \% 0^{5}$ of conjunctival lymphomas are solitary and not associated with systemic disease. In our series, it was seen in patients with a mean age of 45.50 years and twice as commonly in men.

Similar to reports from other countries, such as the USA ${ }^{7}$ and Japan, ${ }^{8}$ most orbital lesions were benign, with hemangiomas and dermoids being the most common. This was followed by benign mixed tumors of the lacrimal gland and meningioma. When meningiomas were subdivided into those of optic nerve origin and those from other surrounding structures, it was noted that optic nerve meningiomas were seen in younger patients, with a shorter clinical course. This is compatible with the findings at the AFIP and can be attributed to the fact that optic nerve meningiomas present with proptosis and visual loss earlier than do meningiomas growing elsewhere. ${ }^{1,2}$

The orbital malignancies can be divided into pediatric and adult categories, with rhabdomyosarcoma being the most common in children, with a mean age of 6.02 years and relatively long mean duration of symptoms ( 7 months). The chances of an orbital tumor being malignant increases with age, and in adults, there is a wider variety of orbital malignancies. The most common is lymphoma $(25.4 \%)$, which was found in the lacrimal gland and orbital soft tissue. Primary lacrimal gland malignancies comprise the next most important group, including adenoid cystic carcinoma (16.2\%), malignant mixed tumor (7.0\%), and adenocarcinoma $(3.5 \%)$, consistent with other reports. ${ }^{7,8}$

Clinically significant is the large number of tumors secondarily invading the orbit from adjacent structures, such as the globe, eyelids, and the sinuses, comprising almost a fifth of all orbital malignancies. This attests to the fact that 
in developing countries like the Philippines, late diagnosis and treatment of cancer is still prevalent and many patients are treated at advanced stages of disease.

The study highlights the tumors that health workers should watch out for. The information presented could be used to guide specialty societies and interest groups in developing early detection programs, and for health insurance agencies to expand treatment packages to include the most important eye cancers.

\section{Conclusion}

We described the distribution and characteristics of all tumors seen for a 10 -year period at the PERI. The majority of lesions from the conjunctiva, eyelids, and orbit were benign, while most intraocular lesions were malignant.

Retinoblastoma was the most common tumor of the eye and ocular adnexa in the series, accounting for $43.2 \%$ of all malignancies. SCC was the most frequent cancer of the conjunctiva, while BCC and SGC were the most common malignancies involving the eyelids. Orbital malignancies had a bimodal distribution, with rhabdomyosarcoma being most common in children, while in adults, lymphoma was most common, followed by lacrimal gland carcinomas.

The study was limited to a review of request forms and histopathologic reports from a single referral center. Clinicopathologic correlations involving staging, treatment, and outcomes are relevant topics for future investigations. A national registry should be established so that epidemiologic data may be obtained from the entire Philippine population and disease patterns observed through time.

\section{Disclosure}

The authors report no conflicts of interest in this work.

\section{References}

1. Ngelangel CA, Wang EH. Cancer and the Philippine Cancer Control Program. Jpn J Clin Oncol. 2002;32 Suppl:S52-S61.

2. Jovanovic P, Mihajlovic M, Djordjevic-Jocic J, Vlajkovic S, Cekic S, Stefanovic V. Ocular melanoma: an overview of the current status. Int J Clin Exp Pathol. 2013;6(7):1230-1244.

3. Lee SB, Au Eong KG, Saw SM, Chan TK, Lee HP. Eye cancer incidence in Singapore. Br J Ophthalmol. 2000;84(7):767-770.

4. Ho M, Liu DTL, Chong KKL, Ng HK, Lam DSC. Eyelid tumors and pseudotumors in Hong Kong: A ten-year experience. Hong Kong Med J. 2013;19(2):150-155.

5. Saornil MA, Becerra E, Méndez MC, Blanco G. [Conjunctival tumors] Arch Soc Esp Oftalmol. 2009;84(1):7-22. Spanish.

6. Gichuhi S, Sagoo MS, Weiss HA, Burton MJ. Epidemiology of ocular surface squamous neoplasia in Africa. Trop Med Int Health. 2013; 18(12):1424-1443.

7. Shields JA, Shields CL, Scartozzi R. Survey of 1,264 patients with orbital tumors and simulating lesions: The 2002 Montgomery Lecture, part 1. Ophthalmology. 2004;111(5):997-1008.

8. Ohtsuka K, Hashimoto M, Suzuki Y. A review of 244 orbital tumors in Japanese patients during a 21-year period: origins and locations. Jpn J Ophthalmol. 2005;49(1):49-55.

9. Bajaj MS, Pushker N, Chaturvedi A, et al. Orbital space-occupying lesions in Indian children. J Pediatr Ophthalmol Strabismus. 2007; 44(2):106-111.

10. Valenzuela RM, Domingo RED, Ranche JM, Manganip LE. A review of pseudoretinoblastoma cases at a tertiary hospital. Philipp J Opthalmol. 2010;35(1):25-31.

11. McLean IW, Burnier MN, Zimmerman LE, Jakobiec FA. Tumors of the eye and ocular adnexa. In: McLean IW, Burnier MN, Zimmerman LE, Jakobiec FA, editors. Atlas of Tumor Pathology. Washington, DC: Armed Forces Institute of Pathology; 1994:161-165.

12. Lee SB, Saw SM, Au Eong KG, Chan TK, Lee HP. Incidence of eyelid cancers in Singapore from 1968 to 1995. Br J Ophthalmol. 1999; 83(5):595-597.

13. Swanson MW, Cloud G. A retrospective analysis of primary eye cancer at the University of Alabama 1958-1988. Part 2: Eyelid tumors. $J$ Am Optom Assoc. 1991;62(11):820-823.

14. Abe M, Ohnishi Y, Hara Y, Shinoda Y, Jingu K. Malignant tumor of the eyelid - clinical survey during 22-year period. Jpn J Ophthalmol. 1983;27(1):175-184.

15. Sihota R, Tandon K, Betharia SM, Arora R. Malignant eyelid tumors in an Indian population. Arch Ophthalmol. 1996;114(1):108-109.

16. Xu XL, Li B, Sun XL, et al. Eyelid neoplasms in the Beijing Tongren Eye Centre between 1997 and 2006. Ophthalmic Surg Lasers Imaging. 2008;39(5):367-372.
Clinical Ophthalmology

\section{Publish your work in this journal}

Clinical Ophthalmology is an international, peer-reviewed journal covering all subspecialties within ophthalmology. Key topics include: Optometry; Visual science; Pharmacology and drug therapy in eye diseases; Basic Sciences; Primary and Secondary eye care; Patient Safety and Quality of Care Improvements. This journal is indexed on

\section{Dovepress}

PubMed Central and CAS, and is the official journal of The Society of Clinical Ophthalmology (SCO). The manuscript management system is completely online and includes a very quick and fair peer-review system, which is all easy to use. Visit http://www.dovepress.com/ testimonials.php to read real quotes from published authors. 